\title{
Quasi-Rayleigh Waves in Butt-Welded Thick Steel Plate
}

\author{
Tuncay Kamas ${ }^{\text {a) }}$ Victor Giurgiutiu ${ }^{\text {b), }}$ Bin Lin $^{\text {c) }}$ \\ a) Mechanical Engineering University of South Carolina \\ 300 Main Str. 29208 Columbia SC \\ ${ }^{b)}$ Mechanical Engineering University of South Carolina \\ 300 Main Str. 29208 Columbia SC \\ ${ }^{c}$ Mechanical Engineering University of South Carolina \\ 300 Main Str. 29208 Columbia SC \\ a)kamas@email.sc.edu \\ b) victorg@sc.edu \\ c)linbin@cec.sc.edu
}

\begin{abstract}
This paper discusses theoretical and experimental analyses of weld guided surface acoustic waves (SAW) through the guided wave propagation (GWP) analyses. The GWP analyses have been carried out by utilizing piezoelectric wafer active sensors (PWAS) for in situ structural inspection of a thick steel plate with butt weld as the weld bead is ground flush. Ultrasonic techniques are commonly used for validation of welded structures in many in-situ monitoring applications, e.g. in off-shore structures, in nuclear and pressure vessel industries and in a range of naval applications. PWAS is recently employed in such ultrasonic applications as a resonator as well as a transducer. QuasiRayleigh waves a.k.a. SAW can be generated in relatively thick isotropic elastic plate having the same phase velocity as Rayleigh waves whereas Rayleigh waves are a high frequency approximation of the first symmetric $\left(\mathrm{S}_{0}\right)$ and antisymmetric $\left(\mathrm{A}_{0}\right)$ Lamb wave modes. As the frequency becomes very high the $\mathrm{S}_{0}$ and the $\mathrm{A}_{0}$ wave speeds coalesce, and both have the same value. This value is exactly the Rayleigh wave speed and becomes constant along the frequency i.e. Rayleigh waves are non-dispersive guided surface acoustic waves. The study is followed with weld-GWP tests through the pitch-catch method along the butt weld line. The tuning curves of quasi-Rayleigh wave are determined to show the tuning and trapping effect of the weld bead that has higher thickness than the adjacent plates on producing a dominant quasi-Rayleigh wave mode. The significant usage of the weld tuned and guided quasi-Rayleigh wave mode is essentially discussed for the applications in the in-situ inspection of relatively thick structures with butt weld such as naval offshore structures. The paper ends with summary, conclusions and suggestions for future work.
\end{abstract}

\section{INTRODUCTION}

Exploring and inventing new structural health monitoring (SHM) technologies enables the industry to reduce the maintenance cost, shorten the service down time, and improve the safety and reliability of engineering structures. SHM methods have improved the management in both the health monitoring of aging structures by predicting the remaining life of the structure and the development of novel self-sensing smart structures by inclusion of sensors. Ultrasonic techniques are commonly used for validation of welded structures in many in-situ monitoring applications: in nuclear industry, in pressure vessel industry, in pipelines, and in a range of naval applications. The tuned quasi-Rayleigh wave mode is essential for the applications in the in-situ inspection of relatively thick structures with butt weld such as naval offshore structures.

The SHM sensors that are capable of active interrogation are called piezo-ceramic wafer active sensors (PWAS). They are widely employed as in-situ ultrasonic health monitoring active sensors in wide frequency band. They can also be used as transmitters that produce traveling waves in a structure so that SHM active and passive sensing can be conducted for interrogation of the structure through wide-band PWAS that generates propagating guided 
ultrasonic waves in substrate structure to detect damages e.g. crack or corrosion by interrogating the structure with certain tuned wave modes ${ }^{1}$.

Rayleigh waves are widely used in non-destructive testing (NDT) and SHM applications as well as in seismology. Rayleigh waves i.e. surface acoustic waves (SAW) are a high frequency approximation of the S0 and A0 Lamb waves as the frequency becomes relatively high, $\mathrm{S} 0$ and the A0 wave speeds coalesce and both have the same value. This value is exactly Rayleigh wave speed. They become non-dispersive wave, i.e. constant wave-speed along the frequency. Rayleigh wave can only travel along a flat surface of a semi-infinite medium, which is hardly possible to generate in reality however for the plate thickness $d>>\lambda_{R}$, the measurements should be acceptable ${ }^{2}$. The wave mode is then called quasi-Rayleigh wave having Rayleigh wave speed. Kamas et-al ${ }^{3}$ discussed the tuning effect of the thickness change and geometry of the substrate material on standing wave modes in local sensing and guided wave modes regarding especially tuned and guided quasi-Rayleigh wave mode propagating in the structure in various geometry and thickness.

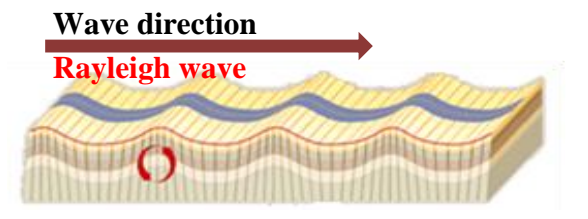

FIGURE 1. Wave-form of the Rayleigh wave on a surface of semi-infinite isotropic elastic medium

In literature regarding weld guided waves, the weld guided compression (S0) mode was first experimentally investigated by Sargent ${ }^{4}$ in butt welded steel plate in $6 \mathrm{~mm}$ thickness for corrosion detection. Juluri ${ }^{5}$ also studied the weld guided S0 and SH0 wave modes using semi-analytical finite element (SAFE) method. They simply simulated the butt-weld bead with thicker region in thickness of $18 \mathrm{~mm}$ and in the width of $16 \mathrm{~mm}$ lying between two steel plate in thickness of $6 \mathrm{~mm}$. The compression mode leaks the SH0 wave in the plates when the velocity of the guided mode in the weld bead is higher than that of SHO wave in the plate. Fan and Lowe $\mathrm{e}^{6}$ carried out an elaborate study and discussed in general the feature guided waves in relatively low frequency range and the physical phenomena of feature guided wave modes.

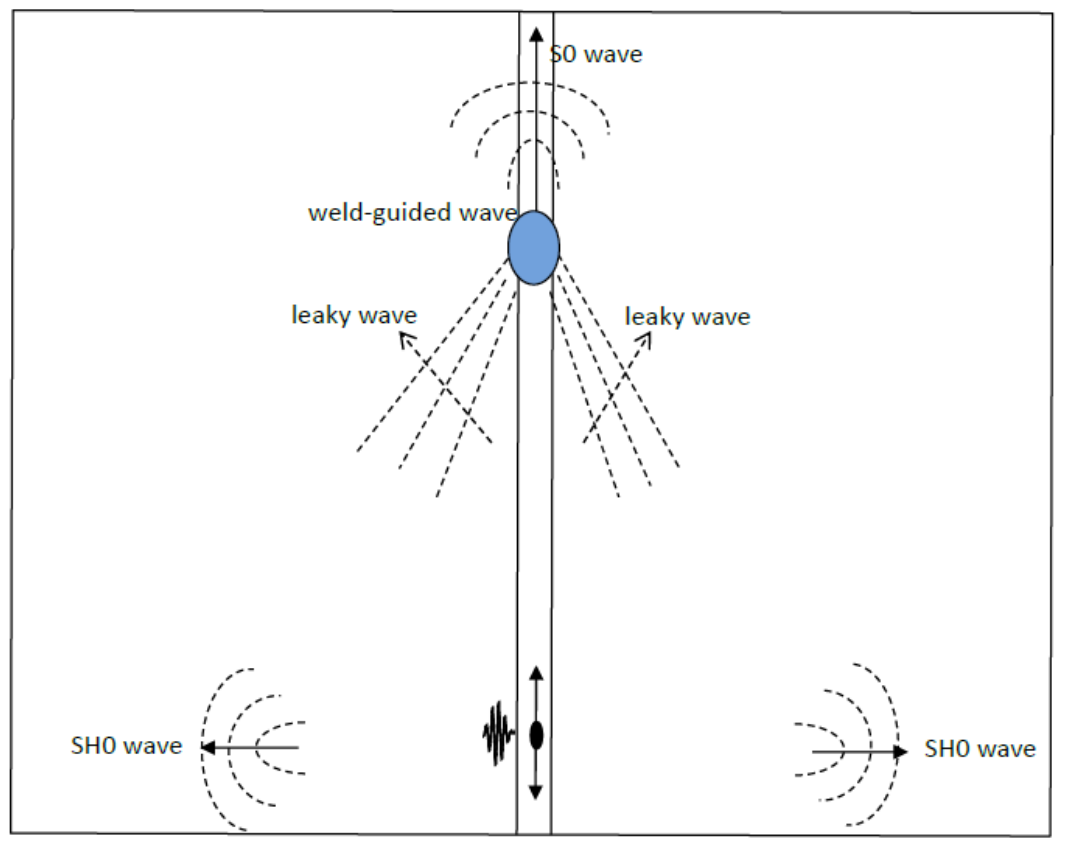

FIGURE 2. Schematic of feature-guided wave propagation on a welded plate

The main objective of this research is to show whether quasi-Rayleigh waves are trapped along a butt weld bead that joints two thick steel plates through the tuning curves from pitch-catch method to investigate the strength of the 
wave mode in weld. The quasi-Rayleigh wave is expected to be received by a receiver sensor that is bonded on lower surface. The quasi-Rayleigh wave-damage interaction is also investigated through finite element models. One eventually can use this SAW method to examine quasi-Rayleigh wave interaction with damages at inaccessible locations in a welded thick plate-like structure.

\section{THEORETICAL ANALYSIS}

Rayleigh waves i.e. surface acoustic waves (SAW) are a high frequency approximation of the fundamental symmetric (S0) and anti-symmetric (A0) Lamb wave modes as the frequency becomes relatively high as seen in FIGURE 3. S0 and A0 wave speeds converges into the same value and become constant i.e. non-dispersive which is exactly the Rayleigh wave speed. Eventhough the condition for Rayleigh wave is that it can only travel along a flat surface of a semi-infinite medium, which is hardly possible to generate in reality, when the plate thickness is larger than the Rayleigh wavelength at that frequency, $d \gg>\lambda_{R}$, the measurements should be acceptable. The wave mode is then called quasi-Rayleigh wave having Rayleigh wave speed. Rayleigh wave speed depends on the shear wave speed and the Poisson ratio. A common approximation of the wave speed of the Rayleigh wave is given in the form

$$
\begin{aligned}
& c_{S}=\sqrt{\frac{E}{2(1+v) \rho}} \\
& c_{R}=c_{S} \frac{(0.87+1.12 v)}{1+v}
\end{aligned}
$$

For more detailed derivation, the readers are suggested to read through chapter 6 of the reference by Giurgiutiu ${ }^{7}$

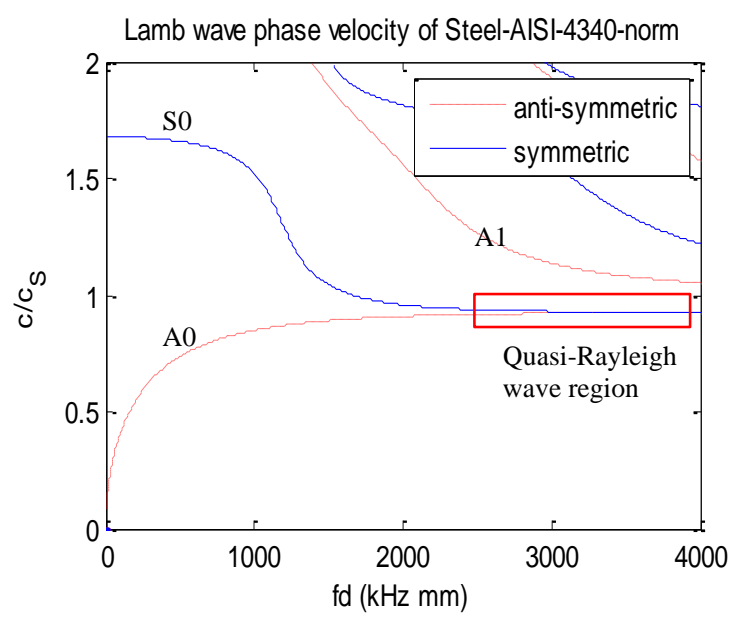

FIGURE 3. Phase velocity dispersion curves of Lamb wave modes in a steel plate where $c_{\mathrm{s}}=3062.9 \mathrm{~m} / \mathrm{s}$ indicating the quasi-

Rayleigh wave approximation of fundamental Lamb wave modes in the region of high frequency-thickness product

The dispersion curves, the displacement vectors, and the mode shapes through thickness are calculated using a MATLAB ${ }^{\circledR}$ graphical user interface (GUI) named 'Modeshape_v2e'8 generated in Laboratory for Active Materials and Smart Structures (LAMSS). In FIGURE 4, the displacement vectors for S0 mode on the left and the S0 mode shape on the right are given as well as in FIGURE 5, the displacement vectors for A0 mode on the left and the A0 mode shape on the right are given. In mode shape across thickness plots shows both the in-plane and out-of-plane displacement changes, the continuous lines depict the in-plane displacement $u_{x}$ and the dotted lines depict the outof-plane displacement $u_{y}{ }^{9}$. 

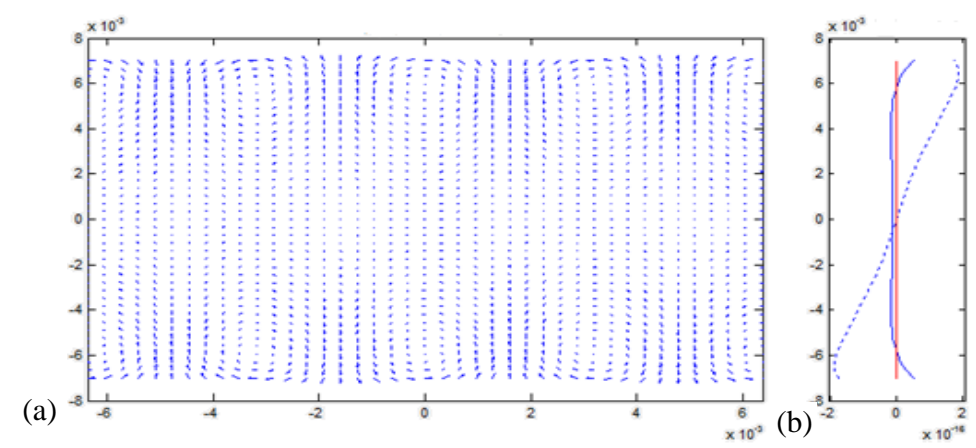

FIGURE 4. (a) Displacement vectors in $14 \mathrm{~mm}$ thick steel plate at central frequency of $450 \mathrm{kHz}$ in the form of the first symmetric Lamb wave mode and (b) the mode shape through thickness

At $f d=3150 \mathrm{kHz} . \mathrm{mm}$, the $u_{x}$ displacement is almost zero over the inner $80 \%$ part of the thickness, while most of the activity happens in the upper and lower $20 \%$ parts; the $u_{y}$ displacement on the surface is almost twice as large as that on the mid-surface FIGURE 5(b).

(a)
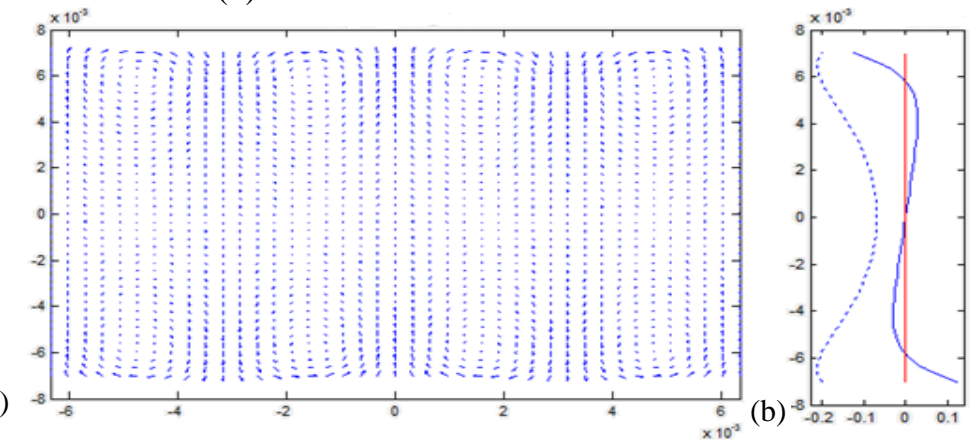

FIGURE 5. (a) Displacement vectors in $14 \mathrm{~mm}$ thick steel plate at central frequency of $450 \mathrm{kHz}$ in the form of the first antisymmetric Lamb wave mode and (b) the mode shape through thickness

The tuning curve of quasi-Rayleigh strain wave (FIGURE 6) is calculated using the strain function which depends on the distance between the transmitter and the receiver PWAS and the expression is shown in $\operatorname{Eq}(2)$.

$$
\varepsilon(x)=i \varepsilon_{a}\left(\sin \xi_{0} a\right) e^{i\left(\xi_{0} x-\omega t\right)}
$$

where $\xi_{0}=\omega^{2} / c_{R}{ }^{2}$ is the wavenumber of Rayleigh waves in the 1-D medium $\varepsilon_{a}=d_{31} E_{3}$ is the induced strain and assumed to be uniform over the PWAS length ${ }^{10}$. The distance between PWAS transducers is measured to be 460 $\mathrm{mm}$. The elastic modulus of steel is assigned to be $190 \mathrm{GPa}$, the density is $7850 \mathrm{~kg} / \mathrm{m}^{3}$, the Poisson ratio is 0.29 . The electrical parameter such as the induced voltage is $80 \mathrm{~V}$ and the piezoelectric parameter $d_{31}$ is $-175 \times 10^{-12} \mathrm{~m} / \mathrm{V}$. The analytical tuning curve agrees well with the experimental tuning curves plots in FIGURE 14.

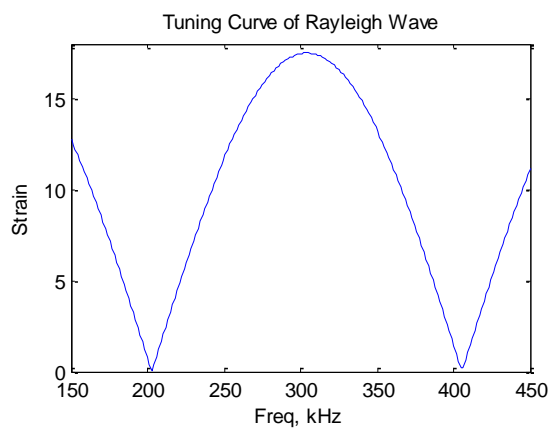

FIGURE 6. Analytical tuning curve of Rayleigh wave in steel plate 


\section{FINITE ELEMENT ANALYSIS}

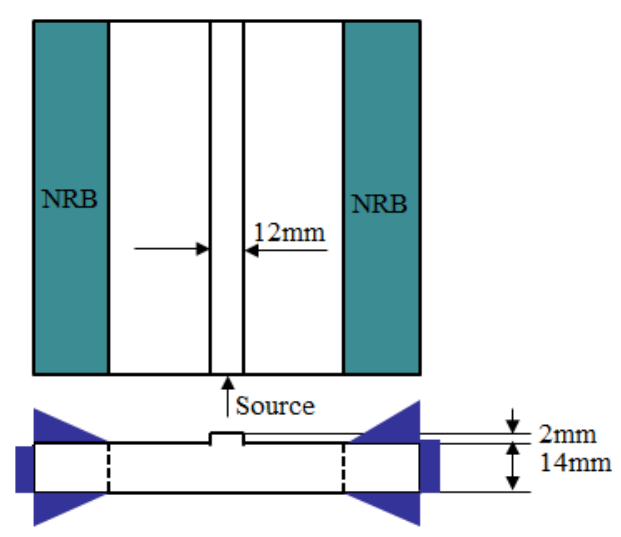

FIGURE 7. Finite element model of butt-welded plate including non-reflecting boundaries on two sides of the steel plate

A square steel plate is modeled in sizes of 400mm x 400mm x 14mm meshed with MESH200 3-D quadrilateral with 8 nodes and SOLID185 3-D 8-node structural solid element. The plate is excited by a pin-point force $\left(\mathrm{F}_{\mathrm{Z}}\right)$ for full-transient analysis on commercial finite element software, ANSYS ${ }$. 400mm x $12 \mathrm{~mm}$ x $16 \mathrm{~mm}$ idealized rectangular butt-weld bead lay out between the adjacent side plates and is meshed with the same solid elements. The material of the weld is assumed to be the same as the plate materials. In addition, non-reflected boundaries (COMBIN14 spring-damper element) were defined with $100 \mathrm{~mm}$ length on two sides as seen in FIGURE 7. The reader is recommended to read through related chapters of the PhD dissertation by Shen ${ }^{11}$ to obtain more detailed information about the non-reflected boundaries.
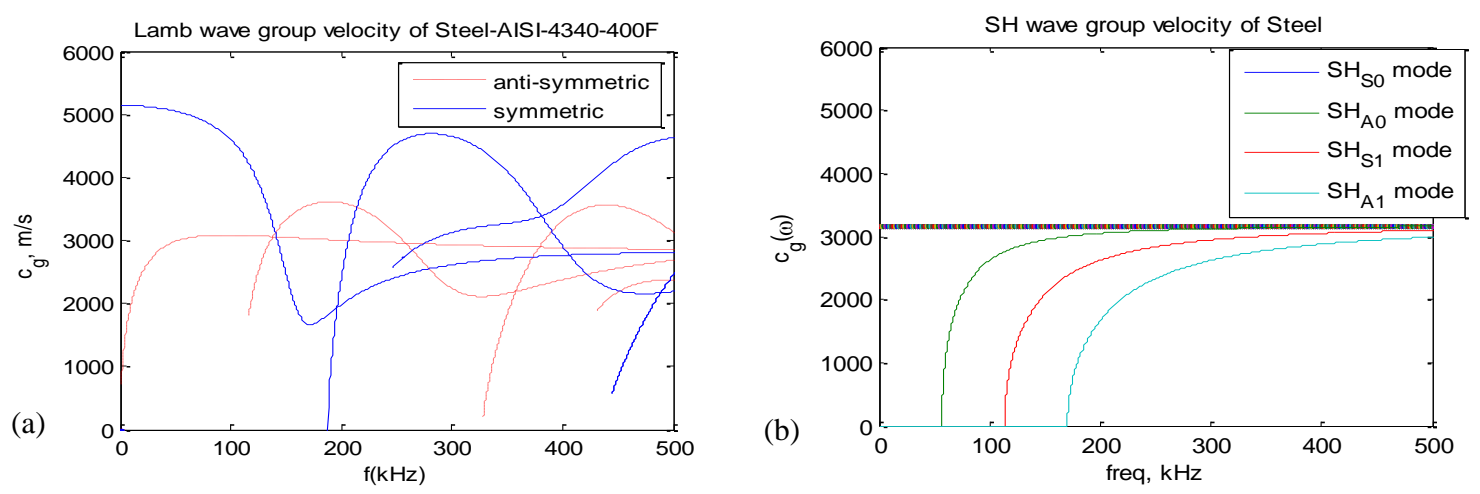

FIGURE 8. Group velocity dispersion curves of (a) Lamb wave modes and (b) shear horizontal (SH) wave modes in steel plate

In FIGURE 8, the group velocities for symmetric and anti-symmetric modes of Lamb waves on the left and of shear horizontal ( $\mathrm{SH}$ ) waves on the right are shown to have track on the weld guided wave modes simulated by the finite element model. Also, the snapshots of animation of the weld guided wave propagation at $67.6 \mu$ sec are illustrated for the pristine and damaged welded plates in FIGURE 9. 


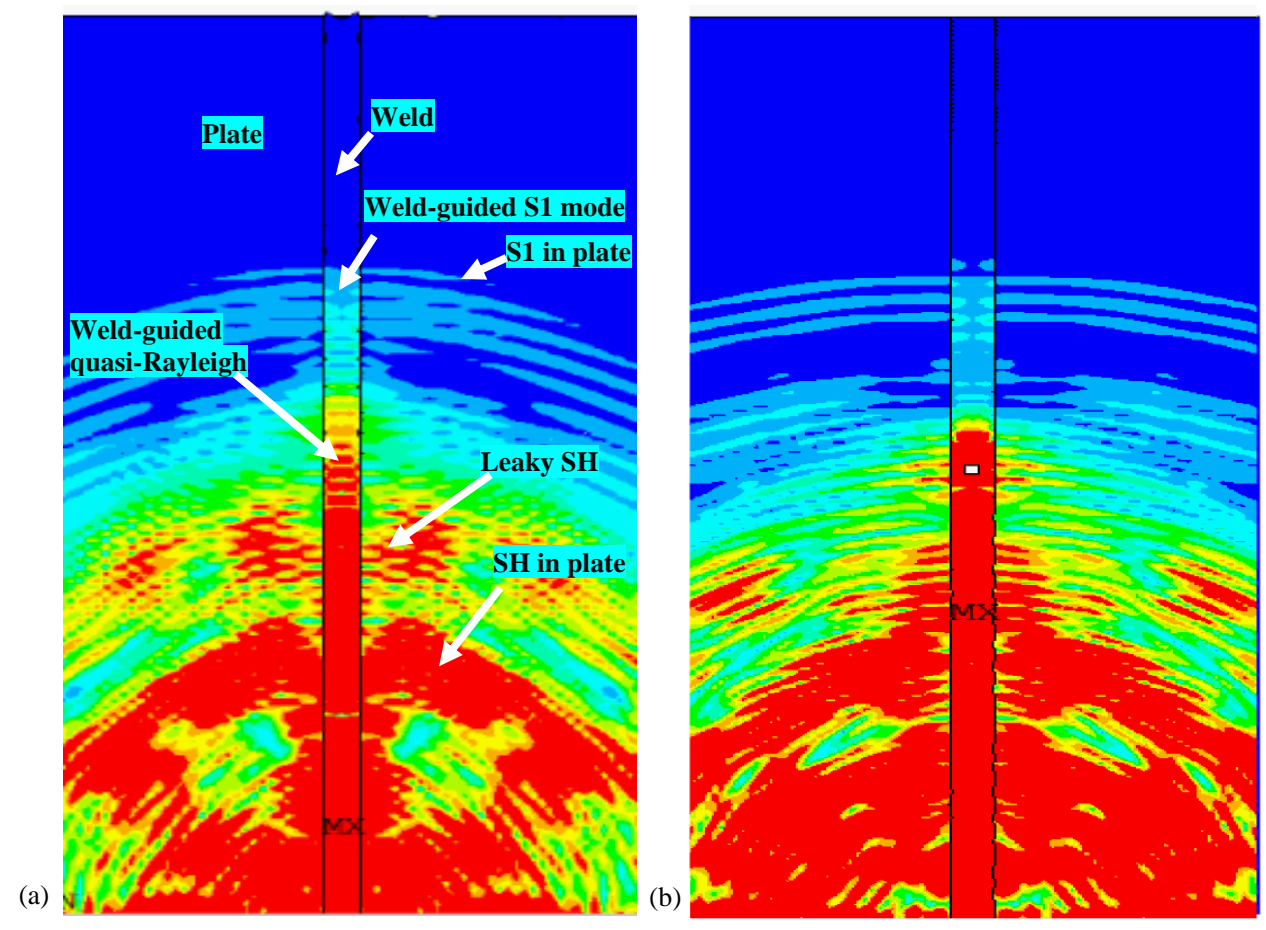

FIGURE 9. Snapshots of weld guided wave simulations on (a) pristine and (b) damaged thick plates. The pin-point force is excited at $300 \mathrm{kHz}$ in both FEA simulations

It is observed from the Lamb wave group velocity dispersion curves that S1 mode is almost one and half time faster than the Rayleigh wave mode in steel plate so that the S1 mode is the leading mode with small amplitude as depicted in the snapshot. The weld guided quasi Rayleigh wave mode follows the S1 mode and leads the leaky SH modes propagating in the wake of the weld guided surface acoustic wave mode toward into the adjacent steel plates. The weld guided wave modes pursue almost the same template in the pristine and damaged plates where the damage is simulated as a hole across the thickness that is introduced in the center of the weld bead. The simulated weld guided wave forms at $300 \mathrm{kHz}$ are demonstrated in FIGURE 10 for the plain (not welded) case as seen on the left and the welded plate case on the right. The results obtained from pristine and damaged plate models are overlapped to understand the response from the weld guided wave-damage interaction on welded thick plate. The same analysis is conducted for the plain plate case so that one can compare the effect of the existence of the weld bead.
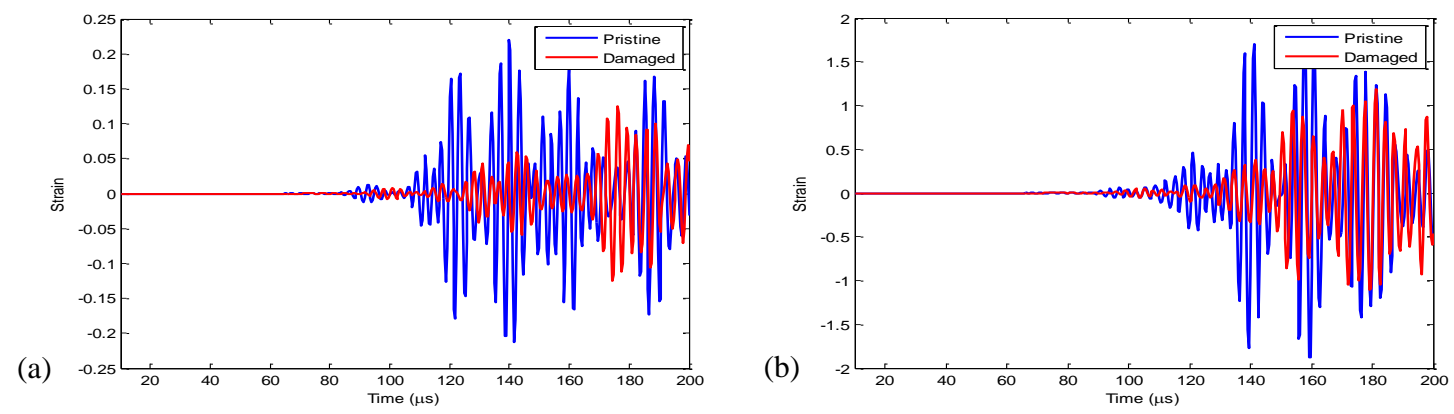

FIGURE 10. Overlapped plots of the received signals from pristine and damaged (a) plain plate and (b) welded plate

As one can clearly observe from the results seen in the plots (FIGURE 10), both weld guided waves and the guided waves in plate reduced in amplitude after they interacted with the damage. However the speed of the waves in weld did not reduce whereas those in the plate reduced. 


\section{EXPERIMENTAL ANALYSIS}

(a)

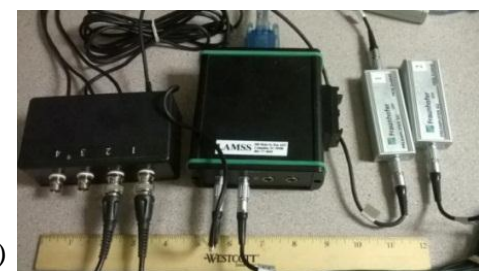

(b) $=$
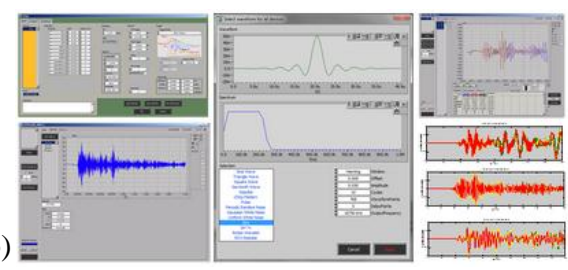

(c)

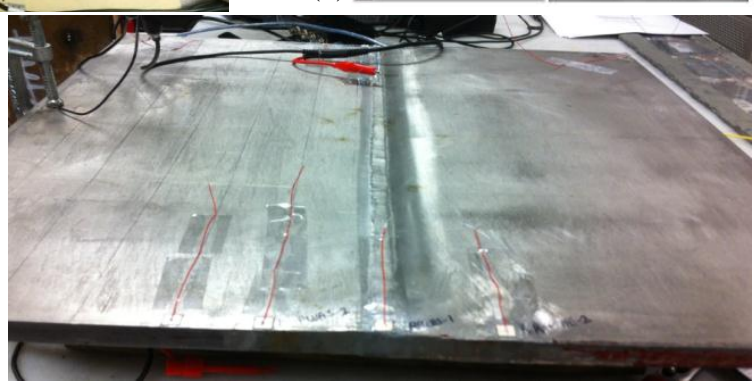

FIGURE 11. (a) multi-channel Acoustic Measurement System (MAS) (b) a LabVIEW graphical user interface, CANWare (c) stainless steel plates in thickness of $14 \mathrm{~mm}$ jointed with butt weld instrumented by $7 \mathrm{~mm} \times 7 \mathrm{~mm} \times 0.2 \mathrm{~mm}$ PWAS

FIGURE 11(c) indicates the specimen employed for the experimental setup. It is stainless steel plates in thickness of $14 \mathrm{~mm}$ jointed with butt weld instrumented by $7 \mathrm{~mm} \times 7 \mathrm{~mm}$ x $0.2 \mathrm{~mm}$ PWAS on and off the weld bead on two ends as the layout can also be seen in FIGURE 12. The excitation signal is selected to be Hanning windowed tone-burst with 5 counts in amplitude of $80 \mathrm{~V}$. For exciting the waves in the substrate structure at transducer terminal and receive the propagating wave signals at the reception terminal, a compact size instrument as seen in FIGURE 11(a) is utilized. The ultrasonic instrument called multi-channel Acoustic Measurement System (MAS) is composed of function generator oscilloscope and preamplifier and designed by Fraunhofer IKTS-MD, Germany. Also a LabVIEW graphical user interface, CANWare as shown in FIGURE 11(b), provided general sensor signal acquisition and basic signal processing for the MAS device.

The layout for the first experimental setup is illustrated in FIGURE 12. We conducted pitch-catch wave propagation technique to acquire the data. The welded steel plate is instrumented by $7 \mathrm{~mm}$ square PWAS transducers on two ends. The first pitch-catch measurement is carried out on the butt-weld and the second one on one of the adjacent steel plates. The path where the wave is generated and received on the weld is called Path-1 and the other is called Path-2.

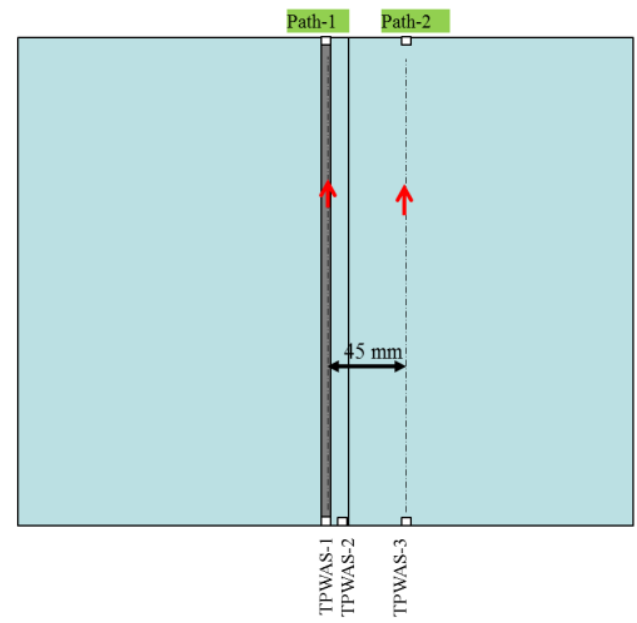

FIGURE 12. Experimental setup layout as stainless steel plates in thickness of $14 \mathrm{~mm}$ jointed with butt weld instrumented by $7 \mathrm{~mm} \times 7 \mathrm{~mm} \times 0.2 \mathrm{~mm}$ PWAS on and off the weld bead on two ends 
The data are acquired for various excitation frequencies in the range between $150 \mathrm{kHz}$ and $450 \mathrm{kHz}$ from both Path. The waveforms for certain frequencies are illustrated in FIGURE 13 to understand when the non-dispersive Rayleigh wave packets (depicted in red boxes) are captured and how large their amplitudes are. Therefore, one can observe and compare the difference of the quasi-Rayleigh wave amplitude acquired from Path-1 and Path- 2 .

\section{Path-1}

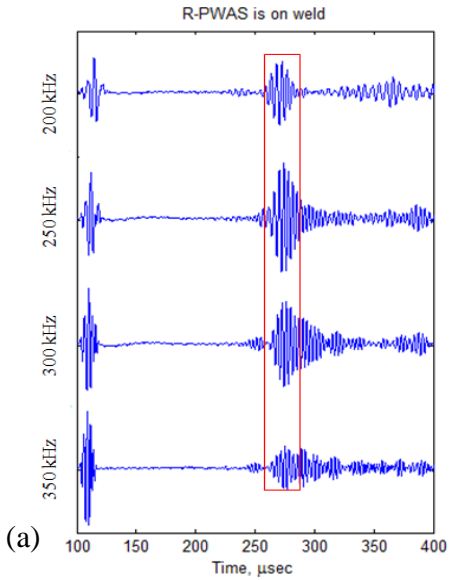

\section{Path-2}

(b)

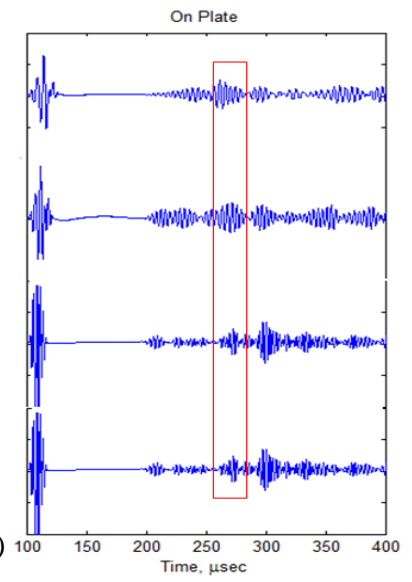

FIGURE 13. Experimental received wave signals at different central frequency from (a) path 1 on weld and (b) path 2 on plate

The amplitude difference is also clearly seen in the experimental tuning curves shown in FIGURE 14. The amplitude of the received quasi-Rayleigh wave packet reaches at its maximum value at around $300 \mathrm{kHz}$ central frequency as predicted by the analytical calculation of the Rayleigh wave tuning curve (FIGURE 6) .As seen in experimental tuning curves, the quasi-Rayleigh wave amplitude reaches up to $3800 \mathrm{mV}$ at the excitation frequency of $270 \mathrm{kHz}$ on Path-1 (on-weld) whereas it is only $2500 \mathrm{mV}$ at the same frequency on Path-2 (off-weld). Hence, the results show that the quasi-Rayleigh wave packet is trapped and guided across the butt-weld bead. Then the quasiRayleigh wave mode can be used as weld-guided wave mode for future applications.
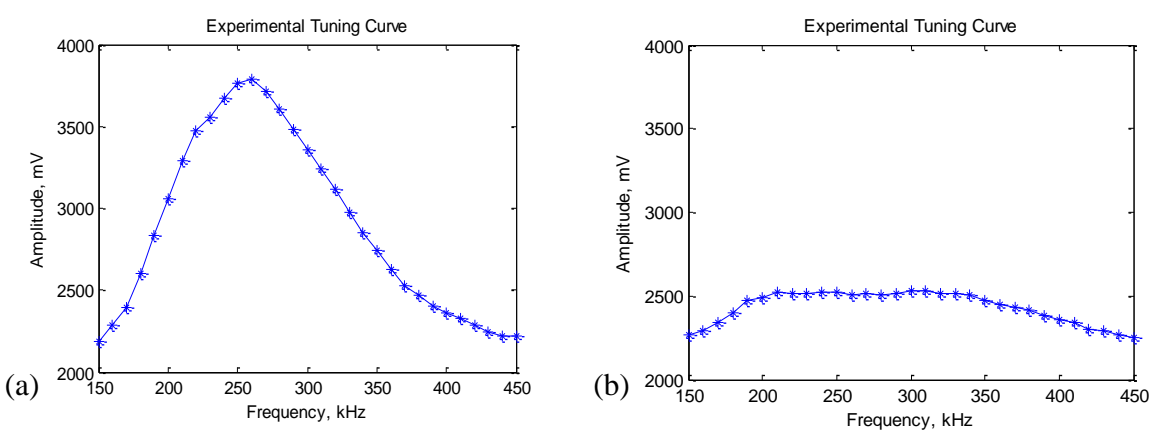

FIGURE 14. Experimental tuning curves of quasi-Rayleigh waves obtained from (a) path 1 on weld and from (b) path2 on plate

The layout for the second set of experiment is illustrated in FIGURE 15. The same butt-welded thick sectioned steel plate is utilized for this experimental setup. As seen, the difference is that the receiver PWAS is attached on the lower surface of the welded thick steel plate whereas the transmitter PWAS is bonded on the upper surface. This GWP pitch-catch technique is conducted to understand how the weld guides the quasi-Rayleigh wave mode. As expected, the wave mode is received with the high amplitude as strong as it was received on the same surface. The mode shape analysis in the previous section (FIGURE 4 and FIGURE 5) also showed the similar perturbation on the lower surface of a plate as the same activity occurs on upper surface at high frequency-thickness product. Thus, this technique can be used to detect a damage that may occur at inaccessible locations on thick plate-like structures. 


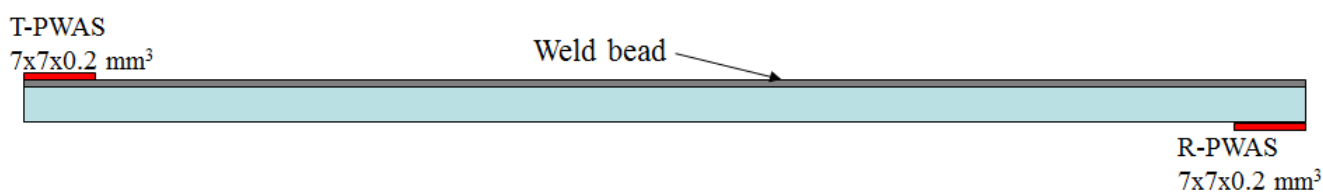

FIGURE 15. Outline of the transmitter and receiver PWAS on the butt-welded stainless steel plate specimen
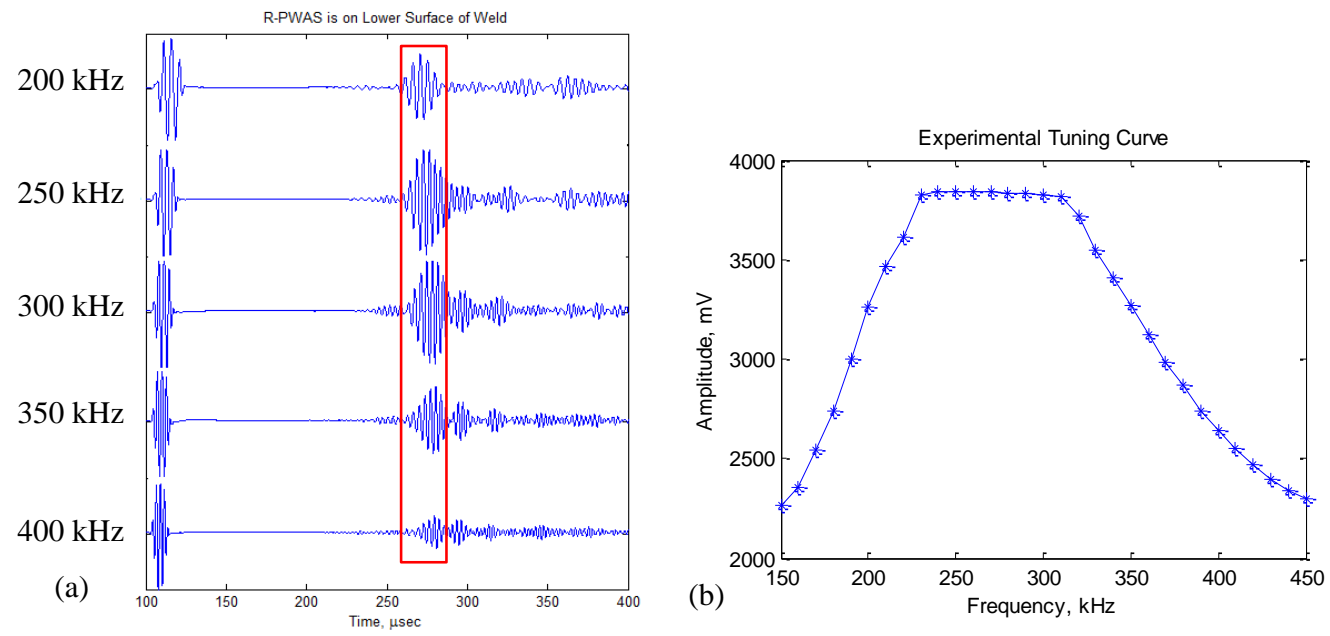

FIGURE 16. (a) Experimental received wave signals at different central frequency (b) Experimental tuning curves of quasiRayleigh waves obtained

\section{SUMMARY AND CONCLUSION}

Experimental and theoretical studies were conducted for in situ structural inspection of a thick steel plate with butt weld. Both experimental and FEA results verified our hypothesis which is the fact that the quasi-Rayleigh wave is guided and tuned by butt-weld having higher amplitude compared to that in the thick plain-plate. We investigated the quasi-Rayleigh wave behavior in pristine structure as well as damaged structure. Experimental and analytical tuning curves agreed. The wave signals and tuning curves showed that quasi-Rayleigh wave traveling in weld can also be received from the opposite surface of a thick plate structure. We theoretically investigated quasi-Rayleigh wave damage interaction by simulated damage modeled as $4 \mathrm{~mm}$ x $4 \mathrm{~mm}$ square hole. Both weld guided waves and waves in plate reduced in amplitude after they interacted with the damage. However the speed of the wave in weld did not reduce whereas that in the plate reduced.

As suggested future work, one can repeat the experiments on thick walled welded pipes to understand how the quasi-Rayleigh wave is captured by R-PWAS installed both on outer and inner surfaces. Eventually, one can investigate the quasi-Rayleigh wave interaction with damages at inaccessible locations in a pipe 


\section{ACKNOWLEDGMENTS}

Support from National Science Foundation Grant \# CMS-0925466; Office of Naval Research \#N00014-11-1-0271, Dr. Ignacio Perez, Program Manager; are thankfully acknowledged.

\section{REFERENCES}

1. V. Giurgiutiu, Structural Health Monitoring with Piezoelectric Wafer Active Sensors (Elsevier Inc., Columbia, SC, USA, 2008), pp. 240-252.

2. M.V. Brook, Ultrasonic Inspection Technology Development and Search Unit Design: Examples of Pratical Applications (IEEE press, 2012), p. 216.

3. T. Kamas, V. Giurgiutiu, and B. Lin, in ASME-SMASIS (2014), pp. 1-9.

4. J. P. Sargent, Insight 48, 160 (2006).

5. N. Juluri, M. Lowe, and P. Cawley, AIP Conference Proceedings 894, 1079 (2007).

6. Z. Fan and M.J.S. Lowe, Proceedings of the Royal Society A: Mathematical, Physical and Engineering Sciences 465, 2053 (2009).

7. V. Giurgiutiu, Structural Health Monitoring with Piezoelectric Wafer Active Sensors, Second Edi (Elsevier Academic Press, Columbia, SC, USA, 2014).

8. J. Bao and V. Giurgiutiu, (2003).

9. J. Bao, Lamb Wave Generation and Detection with Piezoelectric Wafer Active Sensors, University of South Carolina, 2003.

10. V. Giurgiutiu, Structural Health Monitoring with Piezoelectric Wafer Active Sensors (Columbia, SC, USA, 2008).

11. Y. Shen, Structural Health Monitoring Using Linear and Nonlinear Ultrasonic Guided Waves, University of South Carolina, 2014. 